\title{
Pascal et son entourage clermontois : Un acte inédit entre au C.I.B.P.
}

Jean Mesnard et Régine Pouzet

\section{(2) OpenEdition}

\section{Journals}

Édition électronique

URL : http://journals.openedition.org/ccibp/613

DOI : $10.4000 /$ ccibp. 613

ISSN : 2493-7460

Éditeur

Centre international Blaise Pascal

Édition imprimée

Date de publication : 5 février 1992

Pagination : 11-14

ISSN : 0249-6674

\section{Référence électronique}

Jean Mesnard et Régine Pouzet, « Pascal et son entourage clermontois : Un acte inédit entre au

C.I.B.P. », Courrier du Centre international Blaise Pascal [En ligne], 14 | 1992, mis en ligne le 07 janvier 2016, consulté le 01 mai 2019. URL : http://journals.openedition.org/ccibp/613 ; DOI : 10.4000/ ccibp. 613

Ce document a été généré automatiquement le 1 mai 2019.

Centre international Blaise Pascal 


\title{
Pascal et son entourage clermontois : Un acte inédit entre au C.I.B.P.
}

\author{
Jean Mesnard et Régine Pouzet
}

1 Au catalogue d'une superbe vente qui eut lieu à l'hôtel Drouot le 15 mai 1992, figurait (n - 121) ${ }^{1}$ une "signature autographe» de Blaise Pascal, portée au bas d'un contrat d'apprentissage passé à Paris le 27 août 1657. Signature de toute beauté. Le document entier, issu d'une collection anglaise, consistait en une minute notariale de deux pages et demie, remarquablement conservée, montée sur onglet et protégée par une magnifique reliure en maroquin brun à filets dorés. Le contrat - comme le montrait déjà, sur le catalogue, une photographie du dernier feuillet-avait été reçu par le notaire Pierre Parque père (avec, en second, son collègue Renaud Vaultier). Il est évident que la pièce avait été soustraite - à une époque certainement ancienne - dans un fonds passé aujourd'hui au Minutier central des Notaires de Paris². La lacune était impossible à repérer. En effet, quoique Parque père ait exercé de 1612 à 1663, l'inventaire de ses minutes ne conduit que jusqu'en 1651. L'acte mis en vente se trouvait donc, non seulement inédit, mais insoupçonné et insoupçonnable. La révélation était totale.

2 Estimé de 80 à 100000 francs, le document fut adjugé au prix de 140000 francs. Grâce à l'extrême générosité de la Ville de Clermont-Ferrand, appuyée, non moins généreusement, par le Ministère de la Culture, la Bibliothèque municipale et interuniversitaire de la ville a pu faire valoir son droit de préemption : la pièce est venue enrichir somptueusement les collections du Centre International Blaise Pascal.

En voici le texte, transcrit en orthographe moderne, toutes abréviations résolues :

Apprentissage

XXVII août 1657

Fut présent en sa personne Blaise Pascal, écuyer, demeurant à Paris hors la porte SaintMichel, paroisse Saint-Côme, au nom et comme procureur de Monsieur Maître Florin Périer, conseiller en la Cour des Aides de Clermont-Ferrand, tant en son nom propre et privé qu'en qualité de curateur des biens de Jean et Pierre Aubert, ses 
neveux, dudit sieur Périer fondé de procuration spéciale pour l'effet qui ensuit, passée par-devant Maliardon, notaire audit Clermont, en présence de témoins, le dixième jour du présent mois, dont est apparu auxdits notaires soussignés par ladite procuration annexée à ces présentes pour y avoir recours après qu'elle a été paraphée ne varietur par ledit Sieur Pascal et à sa réquisition des notaires soussignés ; lequel sieur Pascal audit nom a reconnu et confessé pour le profit faire et dommage éviter dudit Jean Aubert, âgé de dix-sept ans ou environ, à ce présent, l'avoir baillé et mis en service comme apprenti du jour d'hui jusques à quatre ans prochainement venants et ensuivants à et avec les sieurs Jean Vullart ${ }^{3}$ et Pierre Marsollier, marchands bourgeois de Paris, demeurant rue au Fèvre ${ }^{4}$, paroisse SaintEustache, ledit sieur Vullart à ce présent et acceptant, qui a pris et retenu ledit Jean Aubert, auquel pendant ledit temps il promet montrer et enseigner le trafic et négoce de marchandise et tout ce dont ils se mêlent et entremettent en icelui, lui fournir et livrer les vivres et aliments corporels et le traiter humainement comme il appartient, et ledit apprenti s'entretiendra de tous habits, linge, chaussures et autres ses nécessités convenables à son usage pendant ledit temps; en faveur duquel apprentissage a été accordé entre les parties à la somme de six cents livres, savoir trois cents livres pour la première année et trois cents livres pour la seconde, laquelle somme de trois cents livres pour ladite première année ledit sieur Pascal promet et s'oblige de faire en son propre et privé nom bailler et payer auxdits sieurs Vullart et Marsollier en leur maison à Paris dans quinze jours prochains, et les autres trois cents livres pour ladite seconde année dans dix-huit mois prochainement venants, et pour les deux dernières années ne sera payé aucune chose. Partant ledit apprenti a promis apprendre ledit trafic et négoce de marchandise, servir lesdits maîtres en icelui et en toutes autres affaires qu'ils lui commanderont, faire leur profit, éviter leur dommage et les avertir du contraire s'il vient à sa connaissance, sans s'en défier ne ailleurs servir pendant ledit temps; et en cas d'absence ledit sieur Pascal promet le chercher par les ville et banlieue de Paris, et si trouver le peut le ramener à sesdits maîtres pour parachever le temps susdit, et si icelui sieur Pascal en sondit privé nom certifie ledit apprenti de toute loyauté et fidélité ; car ainsi etc. ; promettant etc. ; obligeant etc.; chacun en droit soi etc. ; ledit apprenti et en corps etc. renonçant etc. Fait et passé ès études desdits notaires le vingt-septième jour d'août mil six cent cinquante sept après midi et ont signé

VULLART PASCAL

AUBERT

Vaultier Parque

En tant que contrat d'apprentissage, cet acte comporte des clauses assurément pittoresques, mais qui ne s'écartent pas des usages habituels en ce domaine. Son intérêt tient surtout au rôle qu'y joue Pascal et aux aspects nouveaux qui se découvrent de ses relations avec les Périer et avec la famille de ceux-ci.

Dans l'admirable suite d'actes notariés relatifs à Pascal et parés de sa signature majestueuse que conserve le Minutier central des Notaires de Paris, en voici donc un nouveau qui en fait implicitement partie. D'autant plus précieux qu'il appartient à une période où de tels actes sont peu nombreux. Un autre porte une date assez proche, la constitution de rente à Port-Royal du 30 juin $1657^{5}$. Mais au-delà, si l'on remonte ou descend le cours du temps, rien ne se trouve après la donation à Florin Périer du $1^{\mathrm{er}}$ octobre $1656^{6}$, ni avant l'inventaire après décès de Louise Delfault commencé le 27 février $1659^{7}$. Le complément ainsi apporté à la connaissance des activités matérielles ou mondaines de Pascal est d'autant plus important qu'il appartient à un registre tout à fait nouveau. 
6 Mais ce qui ressort surtout est d'ordre moral : c'est l'intimité des relations entre Pascal et la famille Périer au long d'une période d'éloignement dont le début coïncide avec le départ de Florin Périer, au terme d'un séjour de quelques mois à Paris, en octobre 1656, et la fin avec l'arrivée de Gilberte dans la capitale, en décembre $1658^{8}$, elle aussi pour un séjour de quelques mois. Dans l'acte, Pascal agit en effet en tant que procureur de Florin Périer, mais on peut constater, aux termes employés, que ce procureur est plus qu'un intermédiaire, qu'il s'engage aussi pour lui-même, qu'il entend agir pleinement en lieu et place de son beau-frère. À titre plus qu'anecdotique, il importe de remarquer que, toujours selon les termes de l'acte, la procuration originale passée à Clermont par Florin Périer, le 10 août, devant son notaire habituel Maliardon, était demeurée attachée à l'acte parisien après avoir été paraphée par Pascal. Or cette pièce annexe fait défaut dans la minute retrouvée. Il est aisé de comprendre pourquoi. En détachant l'annexe, il était possible de mettre dans le commerce une seconde signature de Pascal. Il faut espérer qu'elle se retrouvera un jour.

7 L'acte ne nous apprend rien de nouveau sur l'adresse de Pascal. Le nom du notaire ne tire pas à conséquence: c'est évidemment, à en juger par les adresses, et d'ailleurs conformément aux usages, celui des marchands qui se chargeaient de l'apprentissage, marchands du quartier prospère des Halles et de la rue Saint-Denis. De quel négoce se «mêlaient-ils »? L'absence de précisions suggère qu'il portait sur des marchandises en gros, où draps et tissus devaient tenir une place importante. Le contrat est d'ailleurs expressément passé en vue d'une initiation générale au marketing. Sur les deux associés qui prirent en charge l'apprenti, une recherche particulière serait à mener. Dans l'état actuel de notre information, leurs seuls noms suffisent toutefois à éveiller des échos significatifs. Celui de Vullart, écrit d'abord, dans l'acte, Wulard, évoque invinciblement celui du grand ami de Port-Royal, et, entre autres, de Racine, que fut Germain Vuillart, dont on sait qu'il appartenait à une famille originaire de Flandre, mais établie à Paris depuis deux siècles ${ }^{9}$ il y a beaucoup à parier qu'il était le fils du marchand. Quant au nom de Marsollier, il a été porté par une famille importante dans le milieu dévot et liée à PortRoyal, où plusieurs enfants de ce nom furent élevés aux Petites Écoles ${ }^{10}$. Il n'y a rien de surprenant, tant s'identifient souvent, au XVII siècle, relations d'amitié et relations d'affaires, qu'il y ait lieu de chercher à l'intérieur du cercle des amis de Port-Royal les réseaux par lesquels soit Florin Périer, soit directement Pascal passèrent pour procurer un établissement au candidat apprenti.

Si l'on élargit le commentaire aux dimensions de l'histoire sociale, on observera que, dans les familles tant parisiennes - le cas des Marsollier est exemplaire - que provinciales - l'histoire des Pascal, des Périer et de toute leur parenté en porte témoignage -, les titulaires d'offices de judicature ou de finances côtoient sans cesse les marchands, et que le passage se produit fréquemment d'une situation à l'autre, généralement dans le sens de l'acquisition d'offices, d'autant que par le principe de la vénalité des charges, il fallait, en tout état de cause, posséder d'importants capitaux. L'entrée en apprentissage n'excluait donc pas le désir de satisfaire de hautes ambitions, familiales et personnelles.

9 Reste à faire la connaissance du jeune homme de dix-sept ans dont Pascal assura les débuts dans la vie. Il n'appartenait pas à sa parenté, mais en revanche, et de très près, à celle des Périer. En effet, Marie Périer, la plus jeune sœur de Florin, avait épousé Antoine Aubert et, de ce mariage dont le contrat a été signé le 28 février $1639^{11}$, est né Jean Aubert, baptisé le 7 décembre $1639^{12}$ et un autre fils, Pierre ${ }^{13}$. Le parrain de Jean Aubert est son grand-père maternel, Jean Périer. L'enfant est sans doute élevé à Issoire où son père 
exerce l'office de receveur des tailles. La distance entre Clermont, où la branche maternelle est installée, et Issoire situé à trente-cinq kilomètres au sud, n'entrave pas les relations familiales qui resteront toujours étroites.

10 C'est à l'oncle Florin Périer que l'on fait appel lorsqu'il s'agit de trouver pour le jeune garçon un établissement convenable. Florin toujours prêt à rendre service, qu'il s'agisse de la famille ou des amis, met en œuvre, nous l'avons vu, ses relations parisiennes pour trouver les maîtres d'apprentissage, et utilise ses liens familiaux avec les Pascal pour trouver une caution. Il s'agissait aussi sans doute, en faisant appel à Blaise Pascal, de faciliter l'adaptation du jeune Aubert à son nouveau mode de vie: on le confiait à « un compatriote " déjà bien établi dans la capitale. Ce système "relais", s'agissant des Auvergnats en particulier, fonctionnera jusqu'au début du $\mathrm{XX}^{\mathrm{e}}$ siècle.

11 Malgré ces précautions, Jean Aubert n'accomplit pas le parcours classique des provinciaux qui «montent » à Paris pour tenter d'y faire fortune, et qui y font souche. Son apprentissage dans le «trafic et négoce de marchandise » n'est pas terminé que, le 28 mai 1661, il verse au trésorier des parties casuelles de la reine la taxe qui lui permettra d'exercer la charge de « receveur des épices de MM. les officiers du siège et élection de Clermont-Ferrand ${ }^{14}$ ", office qui a appartenu à son grand-père, Jean Périer, et qui est, depuis le décès de ce dernier, à la fin de l'année 1660 ou au début de l'année 1661, la propriété de Florin Périer. Celui-ci ne pouvant exercer cet office, «à cause de son office de conseiller » et n'ayant pu le vendre «faute d'avoir trouvé de marchands qui en aient voulu donner le juste prix ", a songé à son neveu. Il a fait les démarches pour que le jeune homme qui a maintenant vingt et un ans puisse en être pourvu. Il a assumé également tous les frais des lettres de provision et taxes de réception ${ }^{15}$. Ainsi Jean Aubert reçoit-il ses lettres de provision le 10 juin 1661 . Or le contrat d'apprentissage se terminait le 31 août. Il s'en fallait à peine de trois mois pour qu'il soit honoré. Jean Aubert le dénonce-t-il, l'avait-il déjà dénoncé, est-il toujours au service de Messieurs Vullart et Marsollier pendant qu'on lui prépare à Clermont-Ferrand une nouvelle carrière? Nous ne pouvons le savoir. Ce qui est sûr c'est que Jean Aubert quitte Paris et renonce à la « marchandise ».

Nous le retrouvons à Clermont où il réside désormais, ce qu'attestent divers actes notariés de 1671 et $1672^{16}$, où il apparaît avec les mentions « receveur des épices de MM. du Présidial en cette ville de Clermont y résidant ». Notons qu'il n'est pas propriétaire de l'office. Suivant un usage fréquent à l'époque, Florin lui en a seulement confié l'exercice, et partage avec lui les revenus qui en procèdent. Le jeune homme sans doute n'avait pas les fonds nécessaires à son achat. On note pendant cette période que sa situation n'est pas florissante, et qu'il a toujours recours aux Périer dans les cas difficiles. Ainsi lorsque lui et son frère doivent emprunter 1000 livres à Jacques Pélissier, le 24 janvier 1671, c'est Florin Périer qui sert de caution, et c'est Étienne Périer qui remboursera le créancier en $1678^{17}$. La solidarité familiale joue encore le 7 décembre 1672, quand Gilberte Pascal prête aux deux frères Aubert 600 livres sous forme de contrat de rente ${ }^{18}$.

13 Cependant l'office est réservé à Jean Aubert. Par promesse verbale Florin Périer s'est engagé à le lui vendre à la date du $1^{\mathrm{er}}$ janvier 1672. Florin meurt la même année et c'est Gilberte Pascal qui se charge d'accomplir la promesse. Le 11 avril 1673, pour 6500 livres, elle se démet «de la propriété dudit office de receveur et payeur desdites épices » au profit de Jean Aubert. Tous comptes faits entre les parties, et un legs de Florin à son neveu venant en déduction ainsi qu'une partie de la dot de Marie Périer, Jean Aubert devra 3400 livres à sa tante, qui se réserve de reprendre possession de l'office si le paiement n'est pas effectué. 

Aubert obtient le même jour et devant le même notaire de signer avec sa tante une contre-lettre qui prévoit qu'il sera libre de faire résilier la vente jusqu'au 31 décembre $1676^{19}$. daté du 20 octobre 1677, il n'est plus qualifié payeur des gages et receveur des épices résidant à Clermont, mais "bourgeois habitant de la ville d'Issoire ${ }^{20}$ ». Ce mariage va l'établir ensuite à Bourg-Lastic, petite ville située à une soixantaine de kilomètres à l'ouest de Clermont Ferrand. Son changement de résidence apparaît dans un acte du 8 mars 1681 où l'on voit « $\mathrm{M}^{\mathrm{e}}$ Jean Aubert, bourgeois demeurant à Bourg-Lastic », et son frère Pierre, céder à Gilbert Pignol, moyennant 5000 livres, les droits qui leur reviennent comme héritiers de la dot de leur mère ${ }^{21}$.

Peu de temps après sans doute, Jean Aubert succède à son beau-père, Jacques Mangot, dans ses fonctions de bailli de Préchonnet, fief appartenant à la famille de Langeac, et situé tout près de Bourg-Lastic. Nous le savons grâce au registre paroissial de BourgLastic où figure la mention de son décès : « Le neuvième août 1684 a été enseveli avec les cérémonies de l'église, maître Jehan Aubert, bailli de Préchonnet, étant décédé le septième du présent, muni des sacrements de l'église $[. . .]^{22} »$ Il avait quarante-cinq ans.

Brève carrière, même pour l'époque, et qui, malgré l'assistance familiale, semble avoir été surtout marquée par des échecs ou des abandons. Il laisse une fille prénommée Gilberte, peut-être filleule de Gilberte Pascal, qui deviendra religieuse chez les bénédictines réformées de Clermont-Ferrand dont plusieurs portent les noms de familles amies de Port-Royal ${ }^{23}$.

18 C'est donc bien plus qu'une signature, si belle soit-elle, que nous apporte l'acte heureusement mis à la disposition de tous les amis de Pascal. C'est toute une ouverture sur la vie de Pascal et de sa famille, sur la société clermontoise et auvergnate du XVII ${ }^{\mathrm{e}}$ siècle, sur les relations qu'elle entretenait avec Paris, dans l'ordre des affaires comme dans celui de la vie intellectuelle et spirituelle, sur la portée des liens noués à l'ombre de Port-Royal. Par le biais des réalités quotidiennes du temps, la jonction se fait très vite entre pièces d'archives et textes littéraires.

\section{NOTES}

1. La notice du catalogue comporte quelques erreurs.

2. Étude LXXXVI.

3. Nous suivons l'orthographe de la signature. L'acte porte ici Wulard, et plus bas, Vullard.

4. L'un des noms sous lesquels était désignée la rue plus souvent dite aux Fers.

5. Voir l'édition de Pascal, CEuvres complètes, t. IV, Paris, DDB, 1992, p. 31-34.

6. Ibid., t. III, 1991, p. 954-957.

7. Ibid., t. IV, p. 579-596.

8. Voir les chronologies, ibid., t. III, p. 459 ; t. IV, p. 24.

Courrier du Centre international Blaise Pascal, 14 | 1992 
9. Voir la précieuse édition, par Ruth Clark, des Lettres de Germain Vuillart... à M. Louis de Préfontaine, Genève-Lille, Droz-Giard, 1951, particulièrement p. 9-10.

10. Voir Jean Mesnard, « Familles amies de Port-Royal, II, Les Lombert », Chronique de Port-Royal, $\mathrm{n}$ - 38, 1989, p. 59-61, et les notes, p. 96.

11. Arch. Départ. du P.D.D., 2E01083, (acte du 8 mars 1681).

12. Arch. Départ. du P.D.D., 4F214 (note de l'archiviste Gilbert Rouchon).

13. Le manuscrit de Durand ne distingue pas ces deux fils, voir Pascal, Euvres complètes, t. I, 1964, p. 718.

14. Arch. Départ. du P.D.D., 5E16 DEP 16 (acte du 11 avril 1673).

15. Arch. Départ. du P.D.D., 5E20 DEP 105, (acte du 25 juillet 1663).

16. Arch. Départ. du P.D.D., 5E16 DEP 10, (acte du 24 janvier 1671) ; 5E11 DEP 934, (acte du 7 décembre 1672).

17. Arch. Départ. du P.D.D., 5E16 DEP 10.

18. Arch. Départ. du P.D.D., 5E11 DEP 934.

19. Arch. Départ. du P.D.D., 5E16 DEP 16.

20. Arch. Départ. du P.D.D., 5E37 DEP 831.

21. Arch. Départ. du P.D.D., 2E01083.

22. Arch. Départ. du P.D.D., 6E48/1.

23. Arch. Départ. du P.D.D., 5E11 DEP 34 (acte du 23 février 1716).

\section{INDEX}

Mots-clés : Pascal, acte notarié, CIBP

Keywords : Pascal, notarial deed, CIBP

\section{AUTEURS}

\section{JEAN MESNARD}

Professeur émérite de littérature française du XVIIe siècle Université de Paris-IV

RÉGINE POUZET

CERHAC, Université Blaise Pascal 